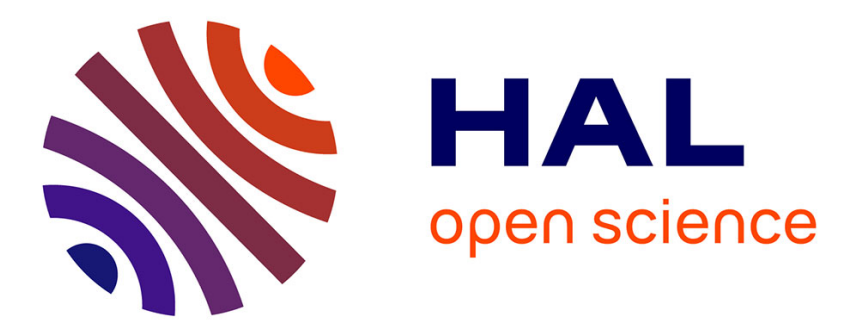

\title{
The modelling of a flap type wave energy converter in a time-dependent mild-slope equation model
}

\author{
Nicolas Tomey-Bozo, Jimmy Murphy, Tony Lewis, Peter Troch, Gareth \\ Thomas, Aurélien Babarit
}

\section{- To cite this version:}

Nicolas Tomey-Bozo, Jimmy Murphy, Tony Lewis, Peter Troch, Gareth Thomas, et al.. The modelling of a flap type wave energy converter in a time-dependent mild-slope equation model. 2nd International Conference on Renewable Energies Offshore (RENEW2016), Oct 2016, Lisbonne, Portugal. 10.1201/9781315229256-35 . hal-01492663

\section{HAL Id: hal-01492663 \\ https://hal.science/hal-01492663}

Submitted on 27 Jun 2019

HAL is a multi-disciplinary open access archive for the deposit and dissemination of scientific research documents, whether they are published or not. The documents may come from teaching and research institutions in France or abroad, or from public or private research centers.
L'archive ouverte pluridisciplinaire HAL, est destinée au dépôt et à la diffusion de documents scientifiques de niveau recherche, publiés ou non, émanant des établissements d'enseignement et de recherche français ou étrangers, des laboratoires publics ou privés. 


\title{
The modelling of a flap type wave energy converter in a time-dependent mild-slope equation model
}

\author{
N. Tomey-Bozo, J. Murphy, T. Lewis \\ Centre for Marine and Renewable Energy (MaREI), University College Cork, Cork, Ireland
}

P. Troch

Department of Civil Engineering, Ghent University, Ghent, Belgium

G. Thomas

Department of Applied Mathematics, University College Cork, Cork, Ireland

\author{
A. Babarit \\ Ecole Centrale de Nantes - CNRS, Nantes, France
}

ABSTRACT: The accurate modelling of the wave field distribution around Wave Energy Convertors (WECs) has been a relevant subject of research in the recent years. Interaction effects in the wave field surrounding the devices are important to know either for power production optimisation of an array or for the quantification of its wake effect due to the absorption of the incoming wave energy. A considerable amount of works have studied arrays of WECs employing methodologies such as BEM based on potential flow theory, wave propagation models, or even CFD in some cases. This study presents an investigation on the modelling of the perturbed wave field by a flap type WEC in a time-dependent mild-slope equation model. The aim of this work is to verify the implementation of a flap in a mild-slope equation model against the wave field obtained from a BEM. Several regular wave cases show the results for different modelling techniques. The capability of modelling the diffracted wave intrinsically in the mild-slope equation model and the implementation of a coupling technique with a BEM are shown. In the last section a representation of the flap by applying the sponge layer technique is done. The understanding of all these techniques with their advantages and disadvantages is relevant for a further development of the wave field modelling. The improvement of the state of the art would allow to quantify more accurately the wake effect of a WEC farm and thus evaluate their capability of acting as a shield with respect to the incoming wave field.

\section{INTRODUCTION}

Several types of models have been employed up to date to quantify the effect of WECs on the incoming wave climate. BEM based on linear waves are the most common application to solve near field effects and farm interactions (Borgarino et al. 2011, McNatt et al. 2014). For large domains an extensive use of wave propagation models has been done using either phased averaged models or phase-resolved models (Smith et al. 2012, Beels et al. 2010a). The usual implementation of these models is undertaken by representing the WECs as sink and sources coefficients or as absorption coefficients where their values are calibrated against a device-dependent data base of reflection and transmission coefficients. CFD models have been applied but are seldom used due to their limitations to modelling very local phenomenon. An overview of this subject can be found in Folley et al. (2012).

As a relatively novel subject of research some studies have developed coupling methodologies in order to improve the accuracy by considering all waves component created by the WEC (Beels 2009, Babarit et al. 2013, Charrayre et al. 2015). The techniques consist of merging the results from BEM solvers into phase-resolved models based on the mild-slope equation. This allows for the combination of the accurate solving at the local scale of the wave-body interaction problem in a BEM with the wave propagation along large domains represented in a mild-slope equation model. Recent applications are capable of solving large grid domains within a relatively low computational time while permitting the representation of irregular bathymetries or dissipation effects such as wave breaking or bottom friction. However up to date only heaving buoys type WECs have been considered which are not the most wave intrusive device.

In this study an investigation on the modelling of a flap type WEC in a time-dependent linear mildslope model (known as MILDwave) for regular waves is shown. The better understanding of the modelling of the wave energy absorbed by a WEC and the disturbed wave field caused by its presence is the motivation of this work. This will allow for further investigations of the quantification of the wake effect produced by a farm of WECs. The core of the work focuses on the implementation of the flap type WEC by applying various techniques and obtaining the perturbed wave field. First the perturbed wave field is calculated by dividing it into the diffracted component and the radiated component. In order to achieve 
this objective a combination of the intrinsic MILDwave feature to solve the diffracted wave and a coupling technique with the open-source BEM solver Nemoh (Babarit, A. \& Delhommeau, G. 2015) to obtain the radiated wave is applied. A comparison with results obtained uniquely from Nemoh are presented.

Then a shorter section shows the implementation of the same flap type WEC employing exclusively MILDwave by applying the sponge layer technique (STL) described in Beels et al. (2010b). This technique consists of a representation of the WEC as a combination of absorption coefficients tuned in such a way that the wave pattern due to reflection and transmission of the wave energy matches specified target values.

\section{ENVIRONMENT DESCRIPTION}

\subsection{Wave-body interaction problem}

Nemoh allows the calculation of the perturbed wave field caused by the presence of the WEC from the perturbed velocity potential obtained by solving the wellknown linear wave-body interaction boundary value problem. The perturbed potential is solved by dividing the problem into one diffraction problem and six radiation problems, one for each degree of freedom. Thus the total velocity potential $\left(\phi_{t}\right)$ can be described as a superposition of the incident potential $\left(\phi_{i}\right)$ and the solution of the diffracted and radiated potential $\left(\phi_{d}\right.$ and $\phi_{r}$ ):

$\phi_{t}(x, y, z)=\phi_{i}+\phi_{d}+\sum_{1}^{6} \phi_{r}$

Then from the potential at the free surface condition the free surface elevation is calculated straightforward as:

$$
\eta(x, y)=\frac{i \omega}{g} \Phi(x, y)
$$

where $\omega$ is the wave frequency, $g$ the gravitational acceleration, and $\Phi$ the potential without depth dependence $(z=0)$.

\subsection{Mild-Slope Equation}

MILDwave was developed at Ghent University by Troch (1998) and is based on the depth-integrated mild-slope equations of Radder and Dingemans (1985). This code solves the propagation of surface waves through the domain and the interaction with the obstacles previously defined. The velocity potential $\Phi(\mathrm{x}, \mathrm{y}, \mathrm{t})$ and the instantaneous surface elevation $\eta(x, y, t)$ at the free water surface are the variables solved for each coordinate of the grid $(\mathrm{x}, \mathrm{y})$ for each instant of time $t$ by the set of differential equations given in Equation (3) and (4):

$$
\frac{\partial \eta}{\partial t}=B \Phi-\nabla \cdot(A \nabla \phi)
$$

$\frac{\partial \Phi}{\partial t}=-g \eta$

where

$$
\begin{aligned}
& B=\frac{\omega^{2}-k^{2} C C_{g}}{g} \\
& A=\frac{C C_{g}}{g}
\end{aligned}
$$

with the phase velocity $C$ and the group velocity $C_{g}$ for a wave with wave number $k$.

\subsection{Flap type wave energy converter}

The WEC is a surface piercing flap hinged at the bottom of the seabed as Figure 1 shows and its main characteristics are described in Table 1.

Table 1. Main characteristics of the flap type WEC.

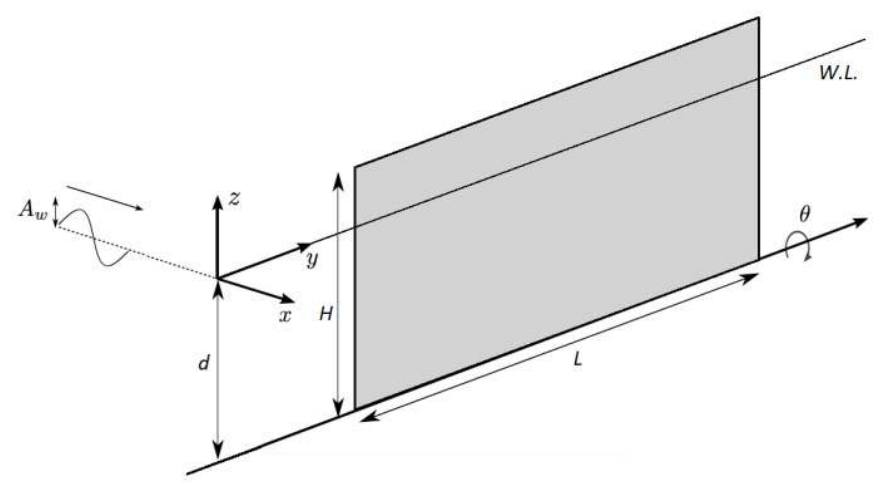

Figure 1. Description sketch of the flap type WEC.

\begin{tabular}{lccc}
\hline Parameter & Coefficient & Value & Units \\
\hline Length & $\mathrm{L}$ & 20 & $\mathrm{~m}$ \\
Height & $\mathrm{H}$ & 12 & $\mathrm{~m}$ \\
Thickness & $\mathrm{t}$ & 1 & $\mathrm{~m}$ \\
Relative density* & $\rho_{\mathrm{r}}$ & 0.3 & - \\
\hline
\end{tabular}

* Density relative to sea water density and assuming a constantly distributed mass.

Its motion is restricted to pitch therefore only one degree of freedom is considered. In order to quantify the non-dimensional radiated wave obtained from Nemoh the motion of the flap is calculated as described in Equation 5:

$\Theta(\omega)=\frac{A_{w} \Gamma(\omega)}{-\omega^{2}(I+A)-i \omega\left(B+B_{p t o}\right)+\left(H+K_{p t o}\right)}$

where $A_{w}$ is the wave amplitude, $\Gamma$ the wave excitation moment coefficient, $I$ the moment of inertia about the $Y$ axis, $A$ the added moment of inertia coefficient, $B$ the radiation damping coefficient, $H$ the hydrostatic restoring coefficient, and $B_{\text {pto }}$ and $\mathrm{K}_{\text {pto }}$ the PTO damping and spring coefficient. The PTO coefficients are tuned for each wave frequency as described in Equation 6 and 7:

$B_{p t o}(\omega)=B(\omega)$ 


$$
K_{p t o}(\omega)=\omega^{2}(I+A)-K
$$

The water depth is considered to be 10 meters and the bathymetry is constant along the whole domain.

\section{WEC MODELLING ACCOUNTING FOR ALL WAVE COMPONENTS}

The perturbed wave field is solved in this section as a sum of the diffraction and radiation components. Only the diffracted wave from the perturbed wave field from a floating body can be intrinsically solved in MILDwave. Thus it is necessary to first show the capability of MILDwave in resolving the diffraction for a surface-piercing flap. Then the radiated wave field is solved in MILDwave by applying the coupling technique with the results obtained from the radiation problem in Nemoh.

All results are compared against the surface elevation obtained from Nemoh and plotted for a domain

of $6 \times 6$ wave lengths. The surface elevation is described by the module and phase from its complex form and it is assumed the incident wave propagates towards the $X$ positive axis. For comparison purposes the surface elevation in MILDwave is saved at a certain instant of time $t$ which is a multiple of the wave period. This allows the comparison to be made with the surface elevation obtained from Nemoh in the frequency domain.

\subsection{Diffracted wave in MILDwave}

The flap is represented in MILDwave as a set of obstacle cells occupying the same length than the device with the absorption coefficient set to 0 (fully reflective obstacle). The diffracted wave is calculated by subtracting the surface elevation obtained in an empty domain (absence of a WEC) to the surface elevation obtained in the presence of the flap.
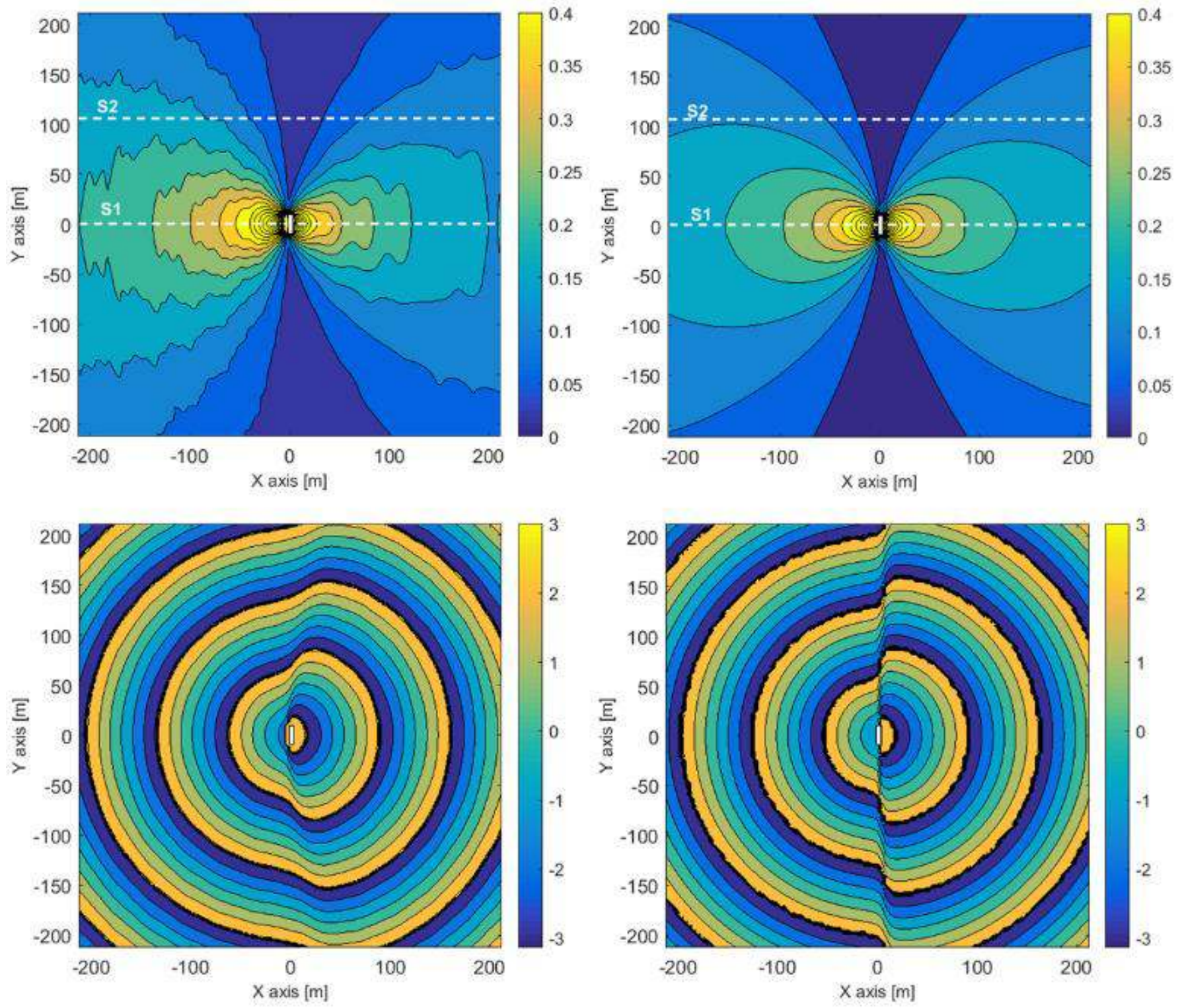

Figure 2. Module (m) and phase (rad) of the complex surface elevation for the diffracted wave. Left figures correspond to results obtained from MILDwave and right figures from Nemoh. 


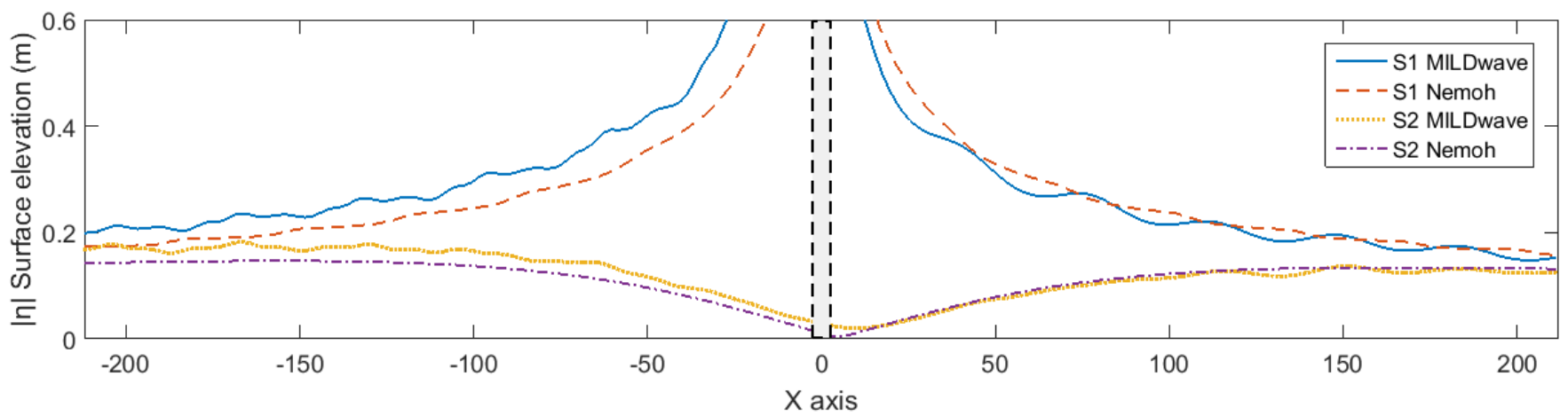

Figure 3. Module of the surface elevation for the diffracted wave for sections S1 and S2 along the X axis showing results obtained from MILDwave and Nemoh.

The diffracted wave field obtained for an incident wave of period $8 \mathrm{~s}$ and unit of amplitude is shown in Figure 2. Left figures are obtained from MILDwave and right figures from Nemoh. The module plots show some discrepancies while the phase plots match entirely. Then Figure 3 shows two sections of the surface elevation module indicated in Figure 2 as S1 and $\mathrm{S} 2$ in order to facilitate the comparison. Higher values for the MILDwave results appear in the upwave field of section S1 due to a larger reflection. However, for the downwave field the matching appears to be better. Section S2 shows a higher agreement for the sides of the domain.

\subsection{Radiated wave with the coupling technique}

The propagation of the radiated wave by the flap under an incident wave of period 8s and unit of amplitude is studied in this section. The radiated wave is defined by imposing an internal circular wave generation line surrounding the device at a defined distance from its centre. Then the wave propagates throughout the domain on the same manner as the radiated wave created by the motion of the device. A definition sketch of the methodology is shown in Figure 4.

The generation line is defined from the complex surface elevation (module and phase) obtained as a solution of the radiation problem from Nemoh at the location of the circular line. In order to obtain the expected propagation of the radiated wave through the domain a set-up of the internal wave generation line needs to be done as described in Beels (2009).

The generation line is defined by a discretisation of grid generation points $i$ determined by a radius dis-

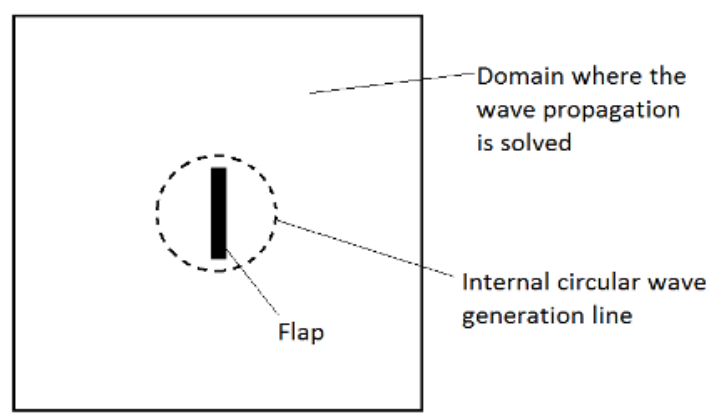

Figure 4. Definition sketch of the coupling technique. tance $r$ to the centre of the domain $(x c, y c)$ and an angle interval $\Delta b$ as presented in Figure 5. In each generation point an additional surface elevation is imposed in MILDwave as defined in Equation (8):

$$
\eta^{*}=2 \eta_{n} \frac{C_{e} \Delta t}{\Delta x}
$$

where $\eta_{n}$ is the surface elevation obtained from Nemoh, $C_{e}$ the energy velocity, $\Delta t$ the time step, and $\Delta x$ the grid cell size.

Taking this into account Figure 6 shows the radiated surface elevation obtained for a wave generation situated at a $20 \mathrm{~m}$ distance from the centre together

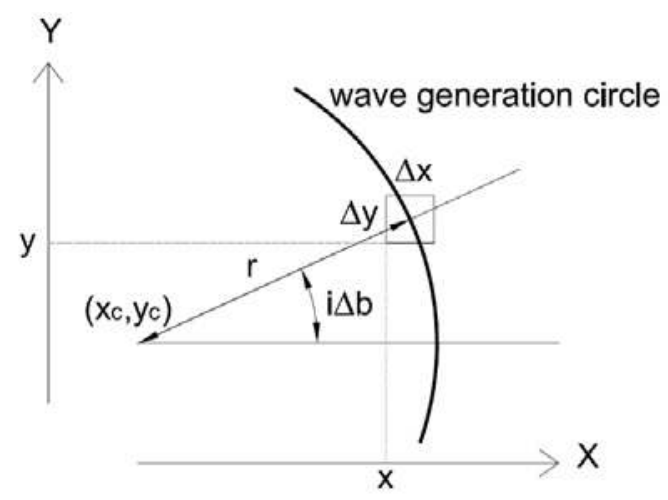

Figure 5. Definition sketch of the internal wave generation line set-up [Beels 2009].

with the surface elevation obtained from Nemoh. Half a wave length distance from the WEC is needed in order to obtain a stabilised wave field, thus the near field is replaced by a circle covering this surface. Both the module and the phase obtained with the coupling technique show accurate results and match entirely the results from Nemoh. In order to facilitate the comparison Figure 7 shows the module of the surface elevation for the two sections indicated in Figure 6 as $\mathrm{S} 1$ and $\mathrm{S} 2$. 

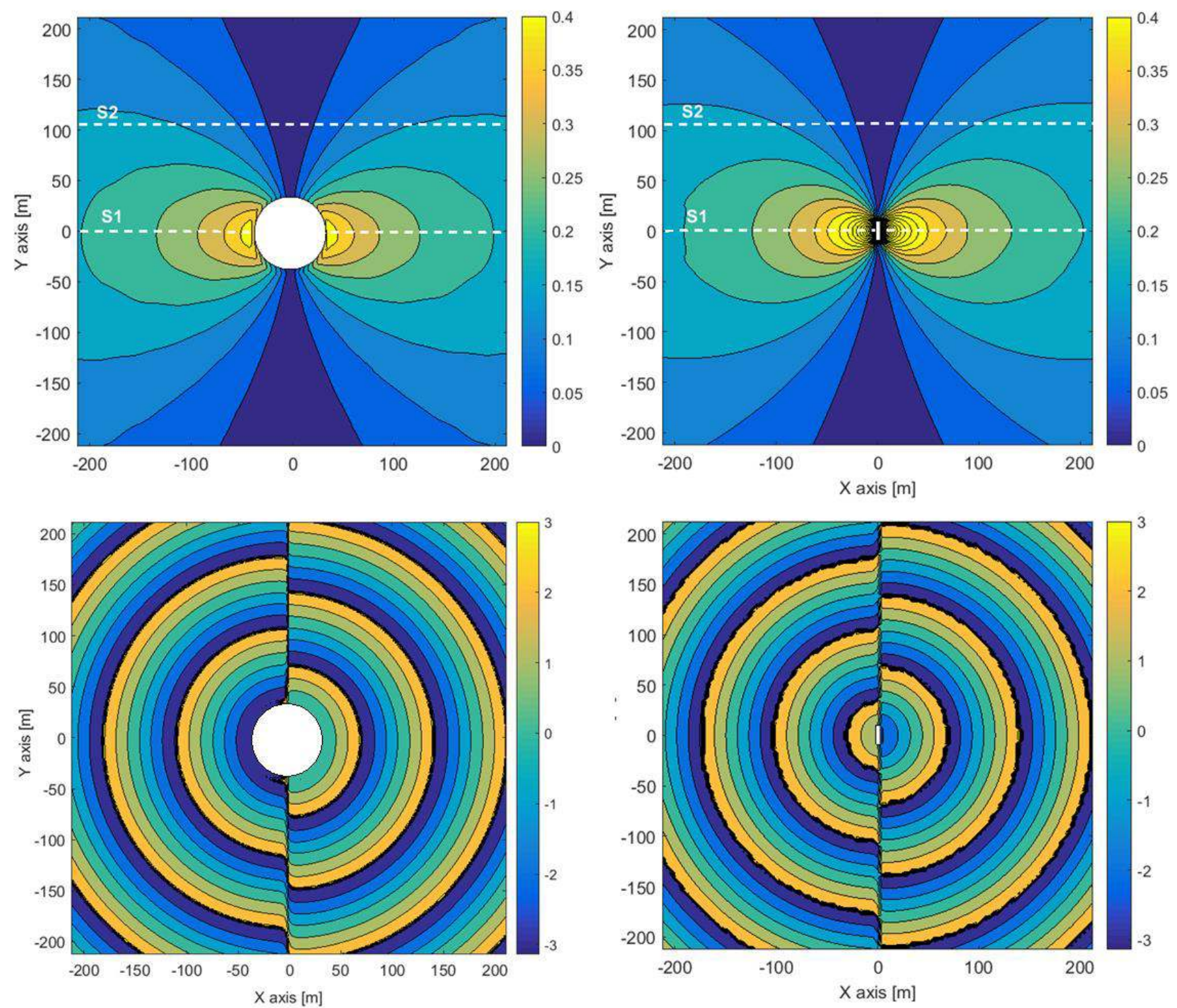

Figure 4. Module (m) and phase (rad) of the complex surface elevation for the radiated wave. Left figures correspond to results obtained from MILDwave employing the coupling technique and right figures to results obtained from Nemoh.

\subsection{Total wave field}

Some limitations can be found under certain circumstances to solve the diffracted wave intrinsically in MILDwave. The representation of the WEC is limited to a set of cells that have a restricted size according to the considered wave length. Thus, depending on the wave and WEC it might be a more suitable option to apply as well the coupling technique to obtain the diffracted wave field.

The surface elevation for the total wave field (incident + diffracted + radiated) has been calculated considering the two different options to solve the diffracted wave, intrinsically in MILDwave and with the coupling technique. Figure 8 shows the module of the complex surface elevation for the total wave field for

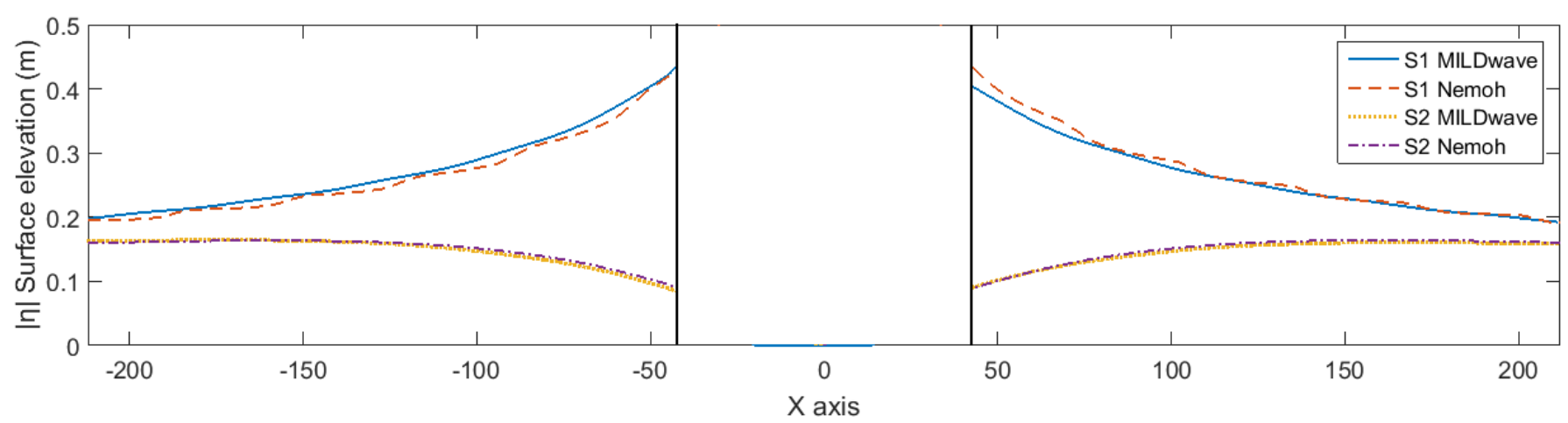

Figure 5. Module of the surface elevation for the radiated wave for sections S1 and S2 along the X axis showing results obtained from MILDwave employing the coupling technique and results obtained from Nemoh. 

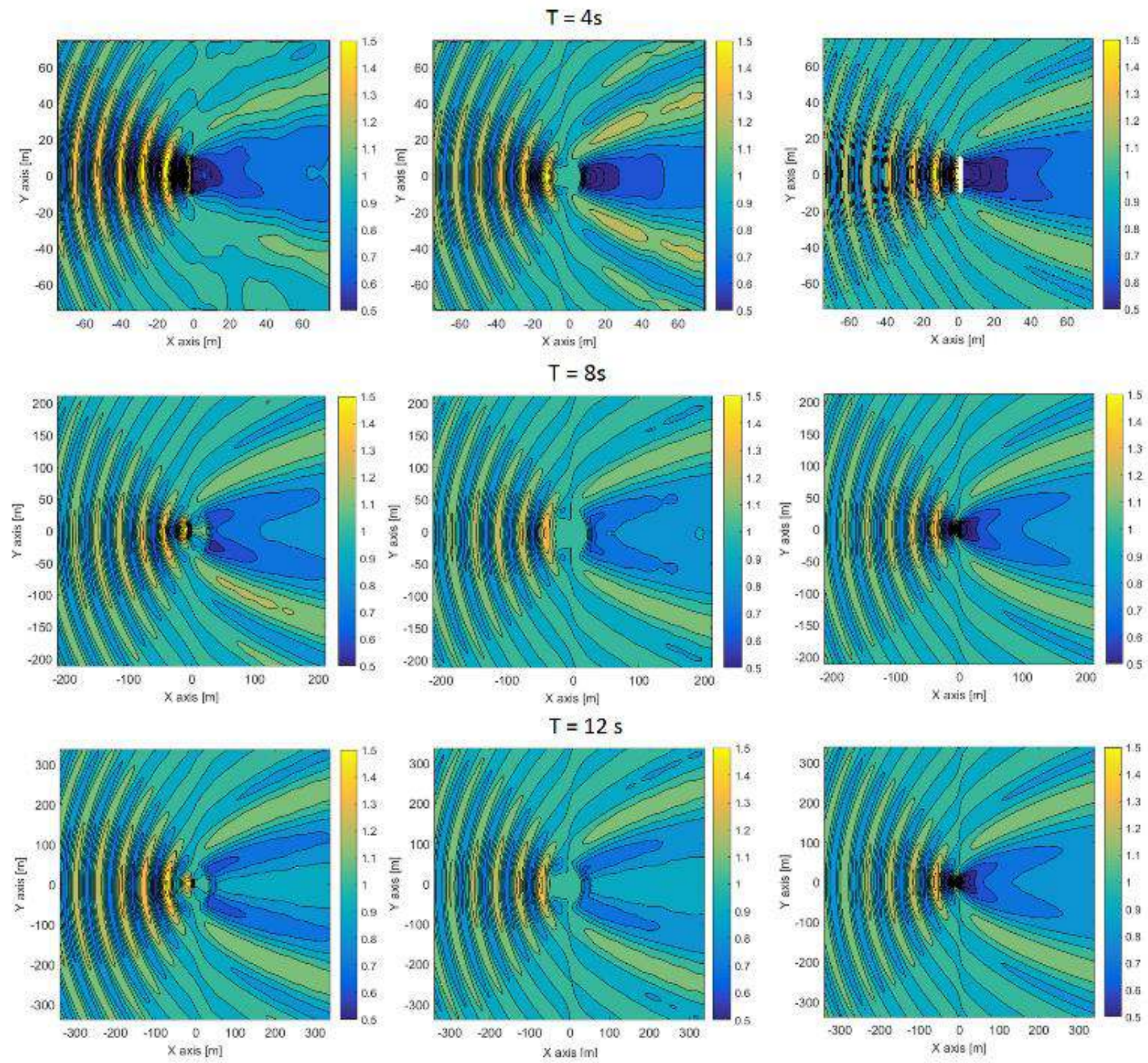

Figure 6. Total wave field for different wave periods $(T)$ accounting for all wave components. Left figures show MILDwave results combining diffraction obtained intrinsically and radiation with the coupling technique. Middle figures show MILDwave results with diffraction and radiation obtained with the coupling technique. Right figures show results obtained from Nemoh.

three different wave periods. Left and middle figures represent the solution obtained with the diffracted wave solved intrinsically in MILDwave and employing the coupling technique respectively. Right figures show the solution from Nemoh as a target for comparison.

It is possible to observe that the two techniques give satisfactory results for all wave periods. Slight discrepancies appear downwave when using the intrinsic feature of MILDwave to solve the diffracted wave. This is partly due to the difficulty of representing the one meter thickness of the flap due to the minimum size of the grid cell.

\section{WEC MODELLING WITH THE SPONGE LAYER TECHNIQUE (SLT)}

The SLT modelling is achieved by representing the presence of the device as a group of obstacle cells that have an attributed absorption coefficient value. The absorption coefficient locally affects the surface elevation of the propagated wave by multiplying it by values in the range from 1 to 0 . Values equal to 1 represents a water cell (no absorption) and then the lower the value the more energy the obstacle absorbs, being 0 a fully reflective obstacle.

The absorption coefficients are tuned in such a way that the perturbed wave field matches the reflection and transmission target values. In this section the target values have been set equal to the disturbed 

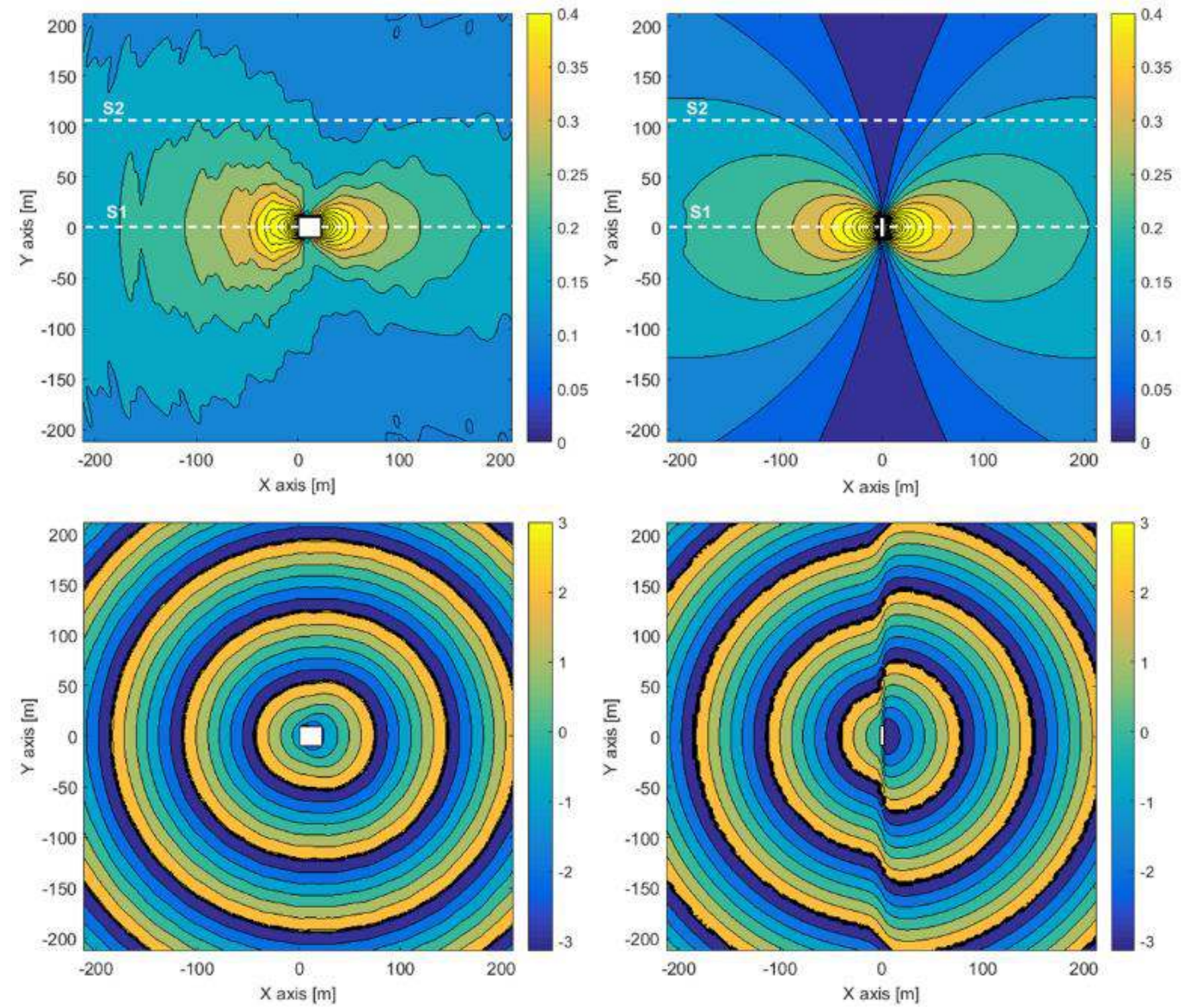

Figure 7. Module (m) and phase (rad) of the complex surface elevation for the perturbed wave. Left figures correspond to results obtained from MILDwave employing the sponge layer technique and right figures results obtained from Nemoh.

wave field obtained from Nemoh in order to evaluate the difference obtained when accounting for all components (as in the previous section) and when applying the SLT.

The set-up of the SLT requires empirical tuning of the obstacle cells values by changing their distribution function. Figure 9 shows the surface elevation for the perturbed wave field created under an incident wave of period $8 \mathrm{~s}$ and unit amplitude. The perturbed wave field is obtained from MILDwave by applying the SLT (for the left figures) and from Nemoh as a sum of the diffraction and radiation component (for the right figures). The MILDwave plots are obtained by subtracting the surface elevation obtained in an empty domain from the surface elevation obtained in the presence of the obstacles cells representing the flap. As a first approximation the tuning has been done to reach the target reflection and transmission values of the perturbed wave field along section S1. Again Figure 10 shows the module of the surface elevation for sections S1 and S2 indicated in Figure 9 in order to facilitate the comparison of results.
It can be observed that the module matches accurately the target values for section S1 but towards the sides of the domain (section S2) more discrepancies are found with larger values obtained in the module when employing the SLT. Then the phase of the surface elevation matches the target in the upwave section while in the downwave section there is a shift of $\pi$ radians. This difference is mainly due to the asymmetry in the motion of the flap which is not considered when applying the SLT.

\section{DISCUSSION}

The surface-piercing flap was considered in order to evaluate the performance of MILDwave to compute the diffracted wave, which is theoretically limited to such devices. Nevertheless a submerged flap can be 


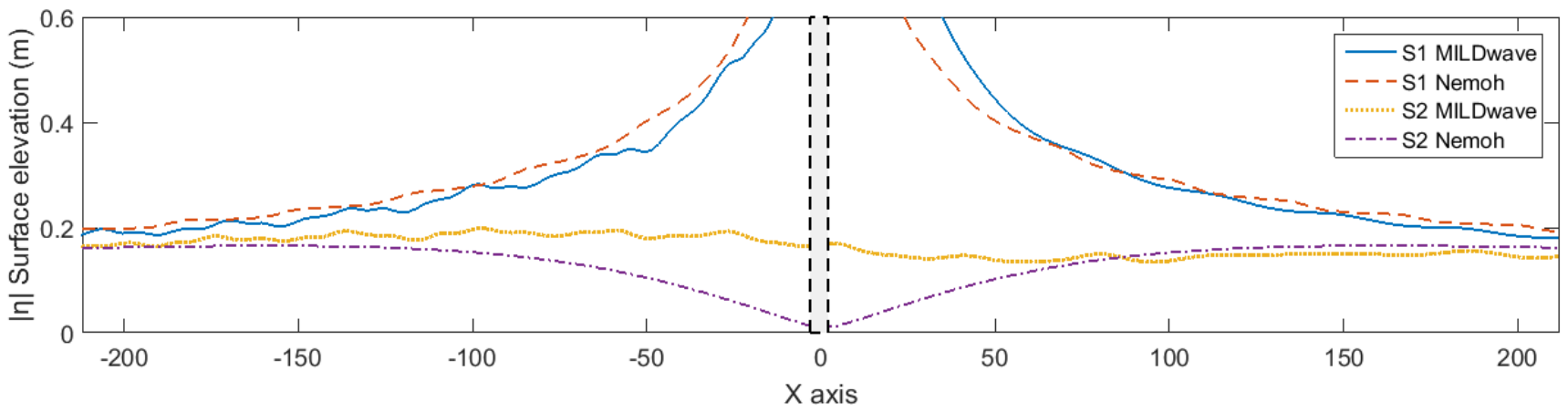

Figure 8. Module of the surface elevation for the perturbed wave for sections S1 and S2 along the X axis showing results obtained from MILDwave employing the sponge layer technique and results obtained from Nemoh.

accurately modelled by solving the complete perturbed wave field applying the coupling technique, in the same way it is was done for the surface-piercing flap.

The coupling technique has shown to provide as accurate results as the ones obtained purely from a BEM. However further work needs to be done to obtain a look up table in order to input the internal wave generation line directly with the right discretization so as to avoid empirical tuning.

The accuracy of the perturbed wave field obtained from the SLT could be improved with further work in the definition of the absorption coefficients and thus achieve results matching completely the module values and differing only in the phase values.

\section{CONCLUSION}

Solving the perturbed wave field accounting for all wave components gives a more realistic definition than applying the SLT, particularly for non-heaving devices where there is half a cycle shift in the phase between the sides of the device. However its application to a farm of several devices and irregular sea states leads to difficult calculations due to the many wave components to consider. Various devices involve accounting for interaction wave components and an irregular sea state for all the present frequencies. An intermediate solution to reduce complexity is to compute all the interaction wave components within the BEM and apply the coupling technique considering the whole farm inside the circular wave generation line.

However, as perturbed waves get dissipated relatively quickly with the distance to the WEC it might be enough to estimate the wake effect with a properly calibrated sponge layer when considering a largely spaced WECs farm. It is still an open subject of discussion as to which values should be taken as a target for the calibration.

\section{ACKNOWLEDGMENT}

The research leading to these results is part of the OceaNET project, which has received funding from the European Union's Seventh Framework Programme for research, technological development and demonstration under grant agreement no 607656.

\section{REFERENCES}

Babarit, A. et al. (2013). On the modelling of WECs in wave models using far field coefficients. Proceedings of EWTEC.

Babarit, A., Delhommeau, G. (2015). Theoretical and numerical aspects of the open source BEM solver NEMOH. Proceedings of EWTEC.

Beels, C. (2009). Optimization of the Lay-Out of a Farm of Wave Energy Converters in the North Sea. Analysis of Wave Power Resources, Wake Effects, Production and Cost. PhD Thesis.

Beels, C. et al. (2010a). Application of the time-dependent mildslope equations for the simulation of wake effects in the lee of a farm of Wave Dragon wave energy converters. Renewable Energy, 35, 1644-1661.

Beels, C. et al. (2010b). Numerical implementation and sensitivity analysis of a wave energy converter in a time-dependent mild-slope equation model. Coastal Engineering, 57, 471492.

Borgarino, B. et al. (2011). Impact of the separating distance between interacting wave energy converters on the overall energy extraction of an array. Proceedings of EWTEC.

Charrayre, F. et al. (2015). A coupled methodology forwavebody interactions at the scale of a farm of wave energy converters including irregular bathymetry. Proceedings of ASME. OMAE2014-23457.

Folley, M. et al. (2012). A Review of Numerical Modelling of Wave Energy Converter Arrays. Proceedings of ASME. OMAE2012-8380.

Mcnatt, J. C., Venugopal, V., \& Forehand, D. (2014). A novel method for deriving the diffraction transfer matrix and its application to multi-body interactions in water waves. Ocean Engineering, 94, 173-185.

Radder, A.C., Dingemans, M.W., 1985. Canonical equations for almost periodic, weakly nonlinear gravity waves. Wave Motion 7, 473-485

Smith, H. C. M. et al. (2012). Further analysis of change in nearshore wave climate due to an offshore wave farm: An enhanced case study for the Wave Hub site. Renewable Energy, 40, 51-64.

Troch, P. (1998). A numerical model for propagation and transformation of linear water waves. Department of Civil Engineering, Ghent University. 